\title{
Uso de plantas medicinais por usuários do serviço público de saúde do município de ljuí/RS
}

\author{
Medicinal plant use by patients using the public health service of the city of ljuí, \\ Rio Grande do Sul, Brazil

\section{El uso de plantas medicinales por los usuarios del servicio público de salud del municipio de ljuí/RS}

Christiane de Fátima Colet. Universidade Regional do Noroeste do Estado do Rio Grande do Sul (UNIJUÍ). ljuí, RS, Brasil. chriscolet@yahoo.com.br (Autora correspondente)

Claudia Angélica Nunes Cavalheiro. Universidade Regional do Noroeste do Estado do Rio Grande do Sul (UNIJUÍ). ljuí, RS, Brasil. claudia@unijui.edu.br

Gislaine Tisott Dal Molin. Universidade Regional do Noroeste do Estado do Rio Grande do Sul (UNIJUÍ). ljuí, RS, Brasil. gmolin@gmail.com Aline Wiliens Cavinatto. Universidade Regional do Noroeste do Estado do Rio Grande do Sul (UNIJUÍ). ljuí, RS, Brasil.

alinecavinatto@yahoo.com.br

Morgana Schiavo. Universidade Regional do Noroeste do Estado do Rio Grande do Sul (UNIJUÍ). ljuí, RS, Brasil. morganaschiavo@yahoo.com.br Karin Hepp Schwambach. Universidade Regional do Noroeste do Estado do Rio Grande do Sul (UNIJUÍ). ljuí, RS, Brasil. karinhepp@yahoo.com.br Karla Renata Oliveira. Universidade Regional do Noroeste do Estado do Rio Grande do Sul (UNIJUÍ). ljuí, RS, Brasil. karla@ unijui.edu.br

\section{Resumo}

Este estudo buscou descrever o uso de plantas medicinais por usuários do Serviço Público de Saúde do município de ljui/RS. Métodos: trata-se de estudo transversal, com questionário estruturado, aplicado aos usuários que buscam atendimento no Serviço Público de Saúde do município de ljuí/RS, nas unidades da Secretaria Municipal de Saúde/ Unidade de Saúde do Centro - UESF -, que atende os bairros Luis Fogliatto e Alvorada, e UESF do Meio-Rural. A coleta de dados ocorreu entre março a setembro de 2012 e foi aprovado pelo CEP/UNIJUí. Resultados: dos 446 entrevistados, $81,0 \%$ utilizam plantas e $71,5 \%$ afirmaram utilizá-las por indicação de familiares. As espécies mais citadas foram: Achyrocline satureioides D.C. (Lam.) (marcela) $22,8 \%$, Matricaria chamomilla L. (camomila) 13,5\%, Lippia alba (Mill.) N.E. Br. ex Britton \& P.Wilson (cidreira) 9,7\%. Quanto às indicações terapêuticas atribuídas às plantas, as mais citadas foram as doenças do trato gastrointestinal, sistema nervoso, distúrbios metabólicos, aparelho respiratório e hipertensão. Algumas destas indicações não encontraram embasamento científico na literatura pesquisada. Conclusão: entende-se necessária a capacitação dos profissionais da área da saúde para garantir a correta orientação ao usuário, bem como o fomento à pesquisa com plantas medicinais para embasar a implementação de políticas públicas de fitoterapia.

\section{Abstract}

This study evaluated the use of medicinal plants by users of the Public Health Service of the city of ljuí, Rio Grande do Sul, Brazil. Methods: this cross-sectional study used a structured questionnaire administered to users who sought care in the Public Health Service of the city of ljuí in units of the Municipal Health Unit/Health Center - UESF serving the Luis Fogliatto, and Alvorada neighborhoods and UESF Meio-Rural. Data collection occurred between March and September 2012 and was approved by the Ethics Research Committee of UNIJUI. Results: of the 446 respondents, $81.0 \%$ used medicinal plants, and $71.5 \%$ said they did so following the advice of family members. The most cited species were Achyrocline satureioides D.C. (Lam.) (marcela), Matricaria chamomilla L. (chamomile), and Lippia alba (Mill.) N.E. Br. ex Britton \& P. Wilson (bushy matgrass), which were used by $22.8 \%, 13.5 \%$, and $9.7 \%$ of the respondents, respectively. The indications most attributed to the plants were diseases of the gastrointestinal tract and nervous system, metabolic disorders, respiratory problems, and hypertension. Some of these indications were not identified by a review of the scientific literature. Conclusion: it is necessary to train health professionals to ensure that correct advice is given to users and to foster research on medicinal plants to support the implementation of public policies on phytotherapy.

Como citar: Colet CR, Carvalheiro CAN, Molin GTD, Cavinatto AW, Schiavo M, Schwambach $\mathrm{KH}$, Oliveira KR. Uso de plantas medicinais por usuários do serviço público de saúde do município de ljuí/RS. 2015;10(36)1-13. http://dx.doi.org/10.5712/rbmfc10(36)930
Palavras-chave:

Plantas Medicinais

Etnobotânica

Atenção Primária à Saúde

Farmácia

Keywords:

Medicinal Plants

Ethnobotany

Primary Health Care

Pharmacies

Fonte de financiamento:

declaram não haver.

Parecer CEP:

UNIJUI, 031/2012, 12/02/2012.

Conflito de interesses:

declaram não haver.

Procedência e revisão por pares: revisado por pares.

Recebido em: 01/05/2014. Aprovado em: 07/08/2015. 


\section{Resumen}

Este estudio describe el uso de plantas medicinales por los usuarios del Servicio de Salud Pública del municipio de ljuí/RS. Métodos: estudio transversal mediante un cuestionario estructurado administrado a los usuarios que buscan atención en el Servicio de Salud Pública del municipio de ljuí/RS, en unidades de la Unidad de Salud/Centro de Salud Municipal - UESF servir barrios Luis Fogliatto, y Dawn y UESF del Medio Rural. La recolección de datos tuvo lugar entre marzo y septiembre del 2012 y fue aprobado por el IRB/UNIJUI. Resultados: de los 446 encuestados, el 81,0\% utilizan plantas y el 71,5\% dijo que utilizan por indicación de la familia. Las especies más frecuentes fueron satureioides Achyrocline (marcela) $22,8 \%$, Matricaria recutita (manzanilla) 13,5\%, Lippia alba (limón) 9,7\%. En cuanto a las indicaciones asignadas a las plantas más citadas fueron: tracto gastrointestinal, el sistema nervioso, trastornos metabólicos, respiratorios, hipertensión y otros. Algunas de estas indicaciones no se encontraron en la literatura científica. Conclusión: se entiende la formación necesaria de los profesionales de la salud para asegurar la orientación correcta para el usuario, así como el fomento de la investigación de plantas medicinales para apoyar a la implementación de políticas públicas en la medicina herbal.

\section{Palabras clave:}

Plantas Medicinales

Etnobotànica

Atencion Primaria de Salud

Farmacia

\section{Introdução}

O uso de plantas medicinais é uma alternativa para a manutenção da saúde, além da prevenção, diagnóstico e tratamento de doenças físicas e mentais. ${ }^{1}$ Estes recursos são uma prática comum nos países em desenvolvimento, nos quais a situação socioeconômica restringe o acesso aos serviços de saúde e o uso tradicional de plantas torna-se, muitas vezes, a única terapia disponível. Além disso, o uso de plantas medicinais está relacionado à valorização da biodiversidade. ${ }^{2}$

Estudos indicam que o cuidado de saúde com produtos a base de plantas medicinais seja favorável à saúde humana, desde que o usuário tenha conhecimento sobre sua finalidade, riscos e benefícios. ${ }^{2}$ A utilização de plantas medicinais pode desencadear efeitos adversos, sendo estes decorrentes de inúmeros fatores, como, por exemplo, falta de conhecimento sobre as condições de cultivo, incorreta identificação farmacobotânica, informações insuficientes sobre reações adversas, esquema posológico, tempo de uso da terapia, e em especial, as interações medicamentosas. ${ }^{3}$

Desta forma, torna-se importante conhecer o perfil de uso de plantas pela população, qualificar o conhecimento da mesma sobre estas terapias e relacionar o conhecimento popular aos estudos científicos, visando estabelecer critérios para o uso seguro de plantas medicinais e fitoterápicos. ${ }^{4}$ Neste contexto, os estudos epidemiológicos sobre o uso das plantas medicinais são importantes para promover o uso racional de medicamentos concomitante com plantas, identificar doenças passíveis de tratamento com estes recursos vegetais e contribuir para a formulação de programas educacionais de saúde que ofereçam informações sobre eficácia, segurança e qualidade de plantas e seus derivados. E, ainda, para auxiliar os profissionais de saúde a incorporar as plantas medicinais nativas como recurso terapêutico do sistema de saúde público. ${ }^{5}$

Assim, o presente estudo teve como objetivo descrever o uso de plantas medicinais por usuários do Serviço Público de Saúde do município de ljuí/RS.

\section{Material e Métodos}

A pesquisa seguiu um modelo de estudo transversal, que utilizou um questionário estruturado, aplicado aos usuários que buscam atendimento no Serviço Público de Saúde do município de ljuí/RS.

ljuí é um município brasileiro localizado na região Noroeste do Estado do Rio Grande do Sul, cuja área de unidade territorial corresponde a $689,133 \mathrm{Km}^{2}$, e população de 78.915 habitantes em $2010 .{ }^{6}$ A Secretaria 
Municipal de Saúde conta com 16 Unidades de Estratégia de Saúde da Família (UESF), distribuídas na área urbana e na área rural.

Os locais de coleta de dados foram selecionados intencionalmente: a) Unidade de Saúde do Centro; b) UESF Distrital, que atende os bairros Luis Fogliatto e Alvorada e c) UESF do Meio-Rural, para representar a população que reside na área urbana/central, urbano-periférica e rural, respectivamente. Embora a Unidade de Saúde do Centro possua no mesmo local um serviço de pronto atendimento, foram selecionados apenas usuários que procuraram atendimento médico nestas Unidades de Saúde selecionadas. Considerando a população atendida neste município foi realizado um cálculo amostral, considerando erro de $5 \%$, e nível de confiança de 95\%, e intervalo de confiança de 95\%, e compôs-se a amostra de 446 entrevistados. Estes foram estratificados nos três locais considerando que a estratificação foi pela proporcionalidade da população cadastrada em cada UBS, da seguinte forma: 297 da Unidade de Saúde do Centro, 74 da UESF do Meio-Rural e 75 da UESF Luis Fogliatto-Alvorada.

Foram incluídos usuários maiores de 18 anos, que procuraram o Serviço Público de Saúde do município de ljuí/RS, nos três locais selecionados para o estudo, no período de março a setembro de 2012 e que aceitaram participar da pesquisa.

A coleta de dados foi realizada com auxílio de um instrumento de coleta de dados, dividido em duas partes, validado em estudo piloto. A primeira parte abrange questões de características socioeconômicas, a segunda refere-se à utilização das plantas, abordando o consumo das mesmas, seu modo de preparo e uso, bem como as suas indicações. Neste momento, a utilização de plantas foi relatada pelos entrevistados de forma verbal. Este estudo faz parte de um projeto de pesquisa que fará a coleta e identificação das plantas em outro momento. Como as coletas foram realizadas em Unidades de Saúde, as plantas utilizadas foram apenas citadas pelos entrevistados, sem coleta do material vegetal neste momento.

Para a questão "finalidade de uso dos chás", foram reunidos termos comuns para as diferentes respostas dos entrevistados e posteriormente agrupados conforme a Classificação Estatística Internacional de Doenças e Problemas Relacionados à Saúde, $10^{\mathrm{a}}$. Revisão (CID 10). ${ }^{7}$ Vale ressaltar que as doenças citadas pelos entrevistados também foram autorreferidas. A análise estatística foi descritiva mediante obtenção de médias e desvios padrão para variáveis quantitativas, com intervalo de confiança de 95\%.

Este estudo é vinculado ao projeto institucional de pesquisa da Universidade Regional do Noroeste do Estado do Rio Grande do Sul - Unijuí intitulado "Avaliação do Uso de Plantas Medicinais e Fitoterápicos no Serviço Público de Saúde do Município de ljuí/RS”, o qual foi aprovado pelo Comitê de Ética da Universidade Regional do Noroeste do Estado do Rio Grande do Sul CEP/UNIJUÍ por meio do Parecer Consubstanciado oㅜ 031/2012.

\section{Resultados}

Foram realizadas 446 entrevistas, destes 79,4\% residiam na área urbana. Destaca-se que 66,6\% foram usuários entrevistados na Unidade de Saúde do Centro, e, destes, 10,4\% informaram residir nesta área do município os demais residiam em regiões periféricas da cidade, mas buscavam atendimento nesta Unidade. Dentre os entrevistados da UESF Luis Fogliatto-Alvorada (distrital), que representaram 16,8\% do total de pessoas, $72,4 \%$ citaram sua moradia nesta área. 
Em relação à faixa etária, a maioria dos usuários (44,6\%) encontra-se entre 40 e 59 anos, com média de idade de 49,75 (DP $\pm 5,75)$. A prevalência de utilização de plantas medicinais foi de $81,0 \%$ entre os entrevistados, sendo sua maioria na faixa etária de 40 a 59 anos (44,6\%). A maioria dos entrevistados relatou possuir ensino fundamental (59\%) e quanto à profissão houve predomínio de do lar $(27,8 \%)$ e aposentados $(14,6 \%)$.

Em relação ao sexo, constatou-se que $87,2 \%$ dos entrevistados eram do sexo feminino e a maior parte dos entrevistados (59,9\%) possui o ensino fundamental completo. Quanto à renda, observou-se que $67,7 \%$ dos usuários apresentam renda de até um salário mínimo e meio e, estratificando-se, observa-se que $75,4 \%$ dos entrevistados da Unidade de Saúde do Centro estão nesta faixa de renda, 67,5\% na UESF Meio Rural e $37,3 \%$ na UESF Luis Fogliatto-Alvorada. Por outro lado, 58,6\% dos usuários entrevistados na UESF Luis Fogliatto-Alvorada apresentam renda de dois a quatro salários mínimos.

Do total de entrevistados, 81,0\% utilizam plantas medicinais na forma de chá. A Tabela 1 caracteriza a utilização de plantas medicinais pelos entrevistados. A forma de utilização e a fonte de indicação dos chás permitiam mais de uma resposta por entrevistado.

A principal forma de uso citada foi a utilização da planta fresca para o preparo do chá, superior a $60 \%$, em todos os locais estudados (Tabela 1). As partes da planta mais utilizada foram as folhas frescas, seguidas pelo uso das flores e caules. A maioria dos entrevistados, $71,5 \%$, mencionou utilizar plantas medicinais por indicação de familiares e 55,3\% afirmaram utilizar plantas medicinais como bebida, portanto, sem fins terapêuticos.

Tabela 1. Caracterização do uso de plantas medicinais na forma de chás por usuários entrevistados de três Unidades de Saúde de ljuí/RS, 2012.

\begin{tabular}{|c|c|c|c|c|}
\hline & $\begin{array}{c}\text { UESF Meio Rural } \\
\text { n (\%) }\end{array}$ & $\begin{array}{l}\text { UESF Luis Fogliatto-Alvorada } \\
\text { n (\%) }\end{array}$ & $\begin{array}{l}\text { Unidade de Saúde do Centro } \\
\text { n (\%) }\end{array}$ & $\begin{array}{l}\text { Total } \\
\text { n (\%) }\end{array}$ \\
\hline Utiliza chá & $n=74$ & $n=75$ & $\mathrm{n}=297$ & $\mathrm{n}=446$ \\
\hline Sim & $65(87,8)$ & $58(77,3)$ & $238(80,1)$ & $361(81,0)$ \\
\hline Não & $09(12,2)$ & $17(22,7)$ & $59(19,9)$ & $85(19,0)$ \\
\hline Como é o chá & $\mathrm{n}=92$ & $\mathrm{n}=83$ & $\mathrm{n}=365$ & $\mathrm{n}=541$ \\
\hline Planta fresca & $64(70,2)$ & $58(67,4)$ & $235(62,1)$ & $357(64,0)$ \\
\hline Sachê & $28(29,8)$ & $25(29,1)$ & $131(34,6)$ & $184(33,0)$ \\
\hline Quem indicou o chá & $n=76$ & $\mathrm{n}=77$ & $\mathrm{n}=318$ & $\mathrm{n}=471$ \\
\hline Família & $62(81,0)$ & $58(75,3)$ & $217(68,2)$ & $337(71,5)$ \\
\hline Amigos & $02(3,0)$ & $04(5,2)$ & $36(11,3)$ & $42(8,9)$ \\
\hline Pastoral da Saúde & $04(5,2)$ & $01(1,3)$ & $21(6,6)$ & $26(5,5)$ \\
\hline Vizinho & $01(1,3)$ & $06(7,8)$ & $13(4,1)$ & $20(4,2)$ \\
\hline Meios de comunicação & $04(5,2)$ & $03(3,9)$ & $11(3,4)$ & $18(3,8)$ \\
\hline Médicos & $02(3,0)$ & $00(0,0)$ & $05(1,7)$ & $07(1,5)$ \\
\hline Farmácia & $01(1,3)$ & $00(0,0)$ & $02(0,6)$ & $03(0,6)$ \\
\hline Outros & $00(0,0)$ & $05(6,5)$ & $13(4,1)$ & $18(4,0)$ \\
\hline
\end{tabular}


As indicações terapêuticas atribuídas às plantas medicinais citadas pelos entrevistados podem ser visualizadas na Tabela 2. As indicações mais prevalentes foram as relacionadas a doenças e sintomas de doenças do trato gastrointestinal, do sistema nervoso, distúrbios metabólicos, doenças do aparelho respiratório, hipertensão arterial sistêmica (HAS), artropatias, doenças infecciosas e parasitárias, diabetes mellitus (DM), doenças do sangue e dos órgãos hematopoiéticos e alguns transtornos imunológicos.

Tabela 2. Plantas medicinais e indicação citadas com maior frequência pelos entrevistados de três Unidades de Saúde de ljuí, $R S / 2012$. $(n=1.359)$

\begin{tabular}{|c|c|c|c|c|c|}
\hline \multirow{2}{*}{$\begin{array}{l}\text { Nome Científico/Nome } \\
\text { Popular }\end{array}$} & \multirow[b]{2}{*}{$\mathrm{n}$ total $(\%)$} & \multicolumn{3}{|c|}{ Indicações mais citadas pelos usuários } & \multirow{2}{*}{$\begin{array}{l}\text { Indicação/Fonte da } \\
\text { indicação }\end{array}$} \\
\hline & & UESF Meio-Rural & $\begin{array}{c}\text { UESF Luis } \\
\text { Fogliatto-Alvorada }\end{array}$ & $\begin{array}{c}\text { Unidade de Saúde do } \\
\text { Centro }\end{array}$ & \\
\hline $\begin{array}{l}\text { Achyrocline } \\
\text { satureioides (Lam.) DC. } \\
\text { (Asteraceae)/Marcela/ } \\
\text { Macela }\end{array}$ & $224(22,8)$ & $\begin{array}{l}\text { Problemas de } \\
\text { estômago, antigripal, } \\
\text { calmante, analgésico } \\
\text { e fígado }\end{array}$ & $\begin{array}{l}\text { Problemas de } \\
\text { estômago, antigripal } \\
\text { e fígado }\end{array}$ & $\begin{array}{l}\text { Problemas de es- } \\
\text { tômago, antigripal, } \\
\text { calmante, analgésico } \\
\text { e fígado }\end{array}$ & 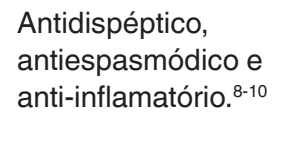 \\
\hline $\begin{array}{l}\text { Matricaria chamomilla L. } \\
\text { (Asteraceae)/Camomila }\end{array}$ & $171(17,4)$ & $\begin{array}{l}\text { Antitérmico, } \\
\text { antitussígeno e } \\
\text { calmante }\end{array}$ & $\begin{array}{l}\text { Calmante, problemas } \\
\text { de estômago, cólica e } \\
\text { analgésico }\end{array}$ & $\begin{array}{l}\text { Calmante, digestivo, } \\
\text { antigripal e analgésico }\end{array}$ & $\begin{array}{l}\text { Uso interno: anties- } \\
\text { pasmódico, ansiolítico } \\
\text { e sedativo leve. } \\
\text { Uso externo: anti-infla- } \\
\text { matório em afecções } \\
\text { da cavidade oral. }{ }^{9-12}\end{array}$ \\
\hline $\begin{array}{l}\text { Lippia alba (Mill.) N.E. } \\
\text { Br. ex Britton \& P. Wilson } \\
\text { (Verbenaceae)/Cidreira }\end{array}$ & $96(9,7)$ & $\begin{array}{l}\text { Calmante, anti- } \\
\text {-hipertensivo, antitér- } \\
\text { mico, analgésico e } \\
\text { antigripal }\end{array}$ & $\begin{array}{l}\text { Calmante, anti-hiper- } \\
\text { tensivo, antitérmico, } \\
\text { antigripal }\end{array}$ & $\begin{array}{l}\text { Calmante, anti-hiper- } \\
\text { tensivo, antitérmico, } \\
\text { analgésico e antigripal }\end{array}$ & $\begin{array}{l}\text { Ansiolítico, sedativo } \\
\text { leve, antiespasmódi- } \\
\text { co e antidispéptico. }\end{array}$ \\
\hline $\begin{array}{l}\text { Plectranthus barbatus } \\
\text { Andrews (Lamiaceae)/ } \\
\text { Boldo nacional, Hortelã } \\
\text { homem, Falso boldo }\end{array}$ & $80(8,1)$ & $\begin{array}{l}\text { Problemas de } \\
\text { estômago, fígado }\end{array}$ & $\begin{array}{l}\text { Problemas de } \\
\text { estômago }\end{array}$ & $\begin{array}{l}\text { Problemas de } \\
\text { estômago, fígado }\end{array}$ & Antidispéptico..$^{9,13-15}$ \\
\hline $\begin{array}{l}\text { Pimpinella anisum L. } \\
\text { (Apiaceae)/Erva doce, } \\
\text { Anis }\end{array}$ & $61(6,2)$ & $\begin{array}{l}\text { Calmante, analgésico, } \\
\text { problemas de } \\
\text { estômago }\end{array}$ & $\begin{array}{l}\text { Calmante, analgé- } \\
\text { sico, problemas de } \\
\text { estômago }\end{array}$ & $\begin{array}{l}\text { Calmante, analgé- } \\
\text { sico, problemas de } \\
\text { estômago }\end{array}$ & $\begin{array}{l}\text { Antidispéptico e } \\
\text { antiespasmódico.9,13 }\end{array}$ \\
\hline $\begin{array}{l}\text { Citrus L. (Rutaceae)/ } \\
\text { Laranjeira }\end{array}$ & $60(6,1)$ & Antigripal, calmante & $\begin{array}{l}\text { Antigripal, calmante, } \\
\text { analgésico }\end{array}$ & Antigripal, calmante & $\begin{array}{l}\text { Ansiolítico e sedativo } \\
\text { leve. } .^{9,13,16,17}\end{array}$ \\
\hline $\begin{array}{l}\text { Mikania glomerata } \\
\text { Spreng. (Asteraceae)/ } \\
\text { Guaco }\end{array}$ & $51(5,2)$ & $\begin{array}{l}\text { Antigripal, } \\
\text { antitussígeno }\end{array}$ & $\begin{array}{l}\text { Antigripal, problemas } \\
\text { de estômago }\end{array}$ & $\begin{array}{l}\text { Antigripal, } \\
\text { antitussígeno }\end{array}$ & Expectorante. $^{9}$ \\
\hline $\begin{array}{l}\text { Mentha L. (Lamiaceae)/ } \\
\text { Hortelã; Hortelã Pimenta }\end{array}$ & $46(4,7)$ & $\begin{array}{l}\text { Antigripal, infecção, } \\
\text { calmante, anti-hiper- } \\
\text { tensivo, digestivo, an- } \\
\text { tioxidante, vermífugo }\end{array}$ & $\begin{array}{l}\text { Antitussígeno, cal- } \\
\text { mante, problemas de } \\
\text { estômago }\end{array}$ & $\begin{array}{l}\text { Antigripal, infecção, } \\
\text { calmante, anti-hiper- } \\
\text { tensivo, digestivo, an- } \\
\text { tioxidante, vermífugo }\end{array}$ & $\begin{array}{l}\text { Antiespasmódico e } \\
\text { antiflatulento. }{ }^{9,13}\end{array}$ \\
\hline $\begin{array}{l}\text { Plantago major L. } \\
\text { (Plantaginaceae)/ } \\
\text { Tanchagem, Tansagem, } \\
\text { Tranchagem }\end{array}$ & $45(4,6)$ & $\begin{array}{l}\text { Antibiótico, problemas } \\
\text { de estômago, anti-in- } \\
\text { flamatório, diabetes, } \\
\text { infecção de bexiga, } \\
\text { purifica o sangue }\end{array}$ & $\begin{array}{l}\text { Antibiótico, infecção } \\
\text { ovariana e útero }\end{array}$ & $\begin{array}{l}\text { Antibiótico, problemas } \\
\text { de estômago, anti-in- } \\
\text { flamatório, diabetes, } \\
\text { infecção de bexiga, } \\
\text { purifica o sangue }\end{array}$ & $\begin{array}{l}\text { Anti-inflamatório } \\
\text { e antisséptico da } \\
\text { cavidade oral. }{ }^{9}\end{array}$ \\
\hline $\begin{array}{l}\text { Malva sylvestris L. } \\
\text { (Malvaceae)/Malva }\end{array}$ & $35(3,5)$ & $\begin{array}{l}\text { Analgésico e anti- } \\
\text {-inflamatório para dor } \\
\text { de garganta e dente }\end{array}$ & $\begin{array}{l}\text { Antibiótico, Analgési- } \\
\text { co e anti-inflamatório } \\
\text { para dor de dente }\end{array}$ & $\begin{array}{l}\text { Analgésico e anti- } \\
\text {-inflamatório para dor } \\
\text { de garganta e dente }\end{array}$ & $\begin{array}{l}\text { Uso interno: } \\
\text { expectorante. } \\
\text { Uso externo: anti- } \\
\text {-inflamatório e antis- } \\
\text { séptico da cavidade } \\
\text { oral. } \text {. }^{9,13,18,19}\end{array}$ \\
\hline
\end{tabular}


Tabela 2. Continuação...

Outras plantas citadas*

490

Aipo/Apium graveolens L. (Apiaceae) (1); Alcachofra/Cynara scolymus L. (Asteraceae) (10); Alecrim/ Rosmarinus officinnalis L. (Lamiaceae) (8); Alho/Allium sativum L. (Amaryllidaceae) (4); Ameixa/ Prunus L. (Rosaceae) (8); Amora branca/Morus alba L. (Moraceae) (3); Aniz estrelado/Illicium verum Hook. f. (Schisandraceae) (11); Arnica/Arnica montana L. (Asteraceae) (2); Arruda/Ruta gravelolens L. (2); Bergamota/Citrus L. (Rutaceae) (14); Cabelo de milho/Zea mays ssp. (Poaceae) (3); Carqueja/Baccharis trimera (Less.) DC. (Asteraceae) (19); Calendula/Calendula officinalis L. (Asteraceae) (2); Canela/Cinnamomum Schaeff. (Lauraceae) (3); Canfora/Cinnamomum camphora (L.); Nees \& Eberm. (Lauraceae) (1); Cáscara sagrada/Rhamnus purshiana DC. (Rhamnaceae) (1); Cavalinha/Equisetum L. (Equisetaceae) (11); Centella asiática/Centella asiatica (L.) Urb. (Apiaceae) (1); Chá preto/Camellia sinensis (L.) Kuntze (Theaceae) (2); Chá verde/Camellia sinensis (L.) Kutze (Theaceae) (15); Chapéu de couro/Echinodorus Rich. (Alismataceae) (11); Cidró/Aloysia citriodora (Paláu) (Verbenaceae) (4); Cipó mil homens/Aristolochia L. (Aristolochiaceae) (9); Cobrina/Peschiera australis (Müll. Arg.) Miers (Apocynaceae) (3); Confrei/Symphytum officinale L. (Boraginaceae)/(4); Couve/Brassica oleraceae L. (Brassicaceae)/(1); Dente de leão/Taraxacum Zinn. (Asteraceae) (1); Endro/Anethum graveolens L. (Apiaceae) (8); Erva de bugre/Casearia sylvestris Sw. (Salicaceae) (4); Espinheira Santa/Maytenus ilicifolia Mart. ex Reissek (Celastraceae) (5); Eucalipto/Eucalyptus L'Her. (Myrtaceae) (1); Funcho/Foeniculum vulgare Mill. (Apiaceae) (14); Gabiroba/Cissus sicyoides fo. lobata (Baker) Planch. (Vitaceae) (14); Zingiber officinale Roscoe (Zingiberaceae)/Gengibre (2); Psidium guajava L. (Myrtaceae)/Goiaba (1); Insulina e Taraxacum F.H. Wigg. (Asteraceae) (5); Ipe/Tabebuia Gomes ex DC. (Bignoniaceae) (1); Jambolão/Syzygium jambolanum (Lam.) DC. (Myrtaceae) (9); Kava-kava/Piper methystichum L. f. (Piperaceae) (1); Lima/Citrus L. (Rutaeae) (1); Limão/Citrus L. (Rutaceae) (21); Losna/Artemisia absinthia St.-Lag (Asteraceae) (8); Maçã/ Malus domestica Borkh.(Rosaceae) (10); Manjericão/Ocimum ser. Basilica Benth. (Lamiaceae) (4); Manjerona/Origanum majorana L. (Lamiaceae) (1); Maracujá/Passiflora sp L. (Passifloraceae) (5); Melissa/Melissa officinalis L. (Lamiaceae) (28); Mestruz/Chenopodium L. (Amaranthaceae) (1); Mil em rama/Achillea millefolium L. (Asteraceae) (1); Morango/Fragaria L. (Rosaceae) (2); Muricata/Annona muricata L. (Annonaceae) (4); Nozes/Carya Nutt. (Juglandaceae) (4); Oliveira/Olea europae L. (Oleaceae) (7); Orégano/Origanum vulgare L. (Lamiaceae) (1); Pata de vaca/Bauhinia forficata Link. (Fabaceae) (1); Pitanga/Eugenia uniflora L. (Myrtaeae) (15); Poejo/Mentha pulegium L. (Lamiaceae) (25); Quebra-pedra/Phyllanthus niruri L. (Phyllanthaceae) (22); Raiz de salsa/ Petroselinum Hill (Apiaceae) (2); Romã/Punica granatum L. (Lythraceae) (6); Sálvia/Salvia officinalis L. (Lamiaceae) (7); Sene/Cassia angustifolia Vahl. (Fabaceae) (3); Sete capota/Campomanesia guazumifolia (Cambess.) O.Berg. (Myrtaceae) (2); Tarumã/Vitex montevidensis Cham. (Lamiaceae) (1); Unha de gato/Uncaria tomentosa (Willd.) DC. (Rubiaceae) (3). Plantas cujo nome cientifico não foi encontrado: Artimege (1); Urtigão (2); Coqueiro vermelho (6); Boldo chinês (1); Infalivina (7); Flor de pedra (1); Frutas vermelhas (2); Erva da pontada (2); Picão do reino (8); Pitoco (1); Juá (2); Penicilina (2); Sucupira (1); Mil ervas (1).

Ocorreram 1.359 citações de plantas medicinais por nome popular, sendo 92 plantas distintas. As plantas citadas com maior frequência estão apresentadas na Tabela 2, com destaque para Achyrocline satureioides D.C. (Lam.) (Asteraceae) (marcela) 22,8\% e Matricaria chamomilla L. (Asteraceae) (camomila) 17,4\%.

Malus domestica Borkh. (maçã), Zea mays ssp. (cabelo de milho), Syzygium jambolanum (Lam.) DC. (jambolão), Campomanesia xanthocarpa Mart. ex O. Berg (gabiroba) e Cissus sicyoides fo. lobata (Baker) Planch. (insulina) encontram-se no Anexo I da RDC 10/10,8 que dispõe sobre a notificação de drogas vegetais junto à Agência Nacional de Vigilância Sanitária (ANVISA) e apresenta uma lista de plantas, com a respectiva forma de uso indicações e contraindicações. No Formulário de Fitoterápicos da Farmacopeia Brasileira, ${ }^{9}$ não constam a Malus domestica Borkh. (maçã), Mentha pulegium L. (poejo), Eugenia uniflora L. (pitanga), Zea mays ssp. (cabelo de milho), Syzygium jambolanum (Lam.) DC (jambolão), Campomanesia xanthocarpa Mart. ex O. Berg (gabiroba) e Cissus sicyoides fo. lobata (Baker) Planch. (insulina).

Quando questionados sobre problemas de saúde, 88,3\% dos entrevistados informaram apresentar pelo menos um, sendo citadas 16 doenças distintas, todas crônicas, com média de três doenças por entrevistado (Tabela 3). Destes, 25,6\% afirmaram ter uma doença, 47,8\% citaram duas doenças e 26,6\%, 
três doenças. Além disso, entre os $11,7 \%$ entrevistados que afirmaram não possuir doenças, $44,7 \%$ relataram não utilizar plantas.

Dentre as doenças crônicas autorreferidas, destaca-se hipertensão arterial sistêmica (HAS) e diabetes mellitus (DM). Do total de entrevistados, $18,5 \%$ se declararam diabéticos e $25,8 \%$ destes usam algum tipo de planta medicinal, como terapia alternativa e complementar para tratarem esta doença. Além disso, $31,8 \%$ dos entrevistados relataram HAS, sendo que mais da metade destes $(53,6 \%)$ fazem uso de algum tipo de planta medicinal, seja para tratamento desta doença, ou para outro fim.

As plantas mais citadas neste estudo para tratamento de HAS foram Lippia alba (Mill.) N.E. Br. ex Britton \& P. Wilson e Mentha L. Já para tratamento de DM, foram citadas Plantago major L., Zea mays ssp., Syzygium jambolanum (Lam.) DC, Campomanesia xanthocarpa Mart. ex O. Berg, Cissus sicyoides fo. lobata (Baker) Planch. e Taraxacum F.H. Wigg.

Além das doenças crônicas acima citadas, 8,0\% dos entrevistados relataram problemas relacionados à depressão. Destes, 28\% afirmaram serem também portadores de DM, HAS, ou ambas, e utilizam plantas medicinais.

\section{DISCUSSÃO}

O uso de plantas medicinais pela população estudada vai ao encontro dos relatos de outros estudos, que demonstraram a larga utilização de plantas medicinais no tratamento de diversas doenças. ${ }^{4}$ No estudo de Oliveira Júnior et al., ${ }^{19}$ realizado em Pernambuco, $72 \%$ relataram utilizar plantas medicinais como medida terapêutica em agravos à saúde. Petry \& Roman-Júnior ${ }^{20}$ verificaram que $98 \%$ dos usuários do SUS entrevistados em Três Passos/RS utilizam plantas medicinais para tratar algum tipo de doença. Lima et al. ${ }^{21}$ verificaram que todos os idosos entrevistados em uma vila periférica de Rio Grande/RS utilizam plantas medicinais no cuidado à saúde, assim como no estudo de Hughes et al., ${ }^{22}$ realizado na África do Sul.

Quanto à parte da planta utilizada, os resultados desse estudo são semelhantes aos de outros. ${ }^{21,23,24}$

Os conhecimentos sobre o uso das plantas medicinais relacionado com a família demonstra que a maior parte deste é transmitido de geração para geração. ${ }^{24}$ Este fato também foi registrado no estudo

Tabela 3. Doenças citadas pelos usuários de plantas medicinais entrevistados de três Unidades de Saúde de ljuí/RS, 2012. (n=481)

\begin{tabular}{|c|c|c|c|c|}
\hline \multirow[t]{2}{*}{ Doenças } & $\begin{array}{l}\text { UESF Meio-Rural } \\
\qquad \mathrm{n}=81\end{array}$ & $\begin{array}{l}\text { UESF Luis } \\
\text { Fogliatto-Alvorada } n=79\end{array}$ & $\begin{array}{l}\text { Unidade de Saúde } \\
\text { do Centro } n=321\end{array}$ & $\begin{array}{c}\text { Total UESF } \\
n=481\end{array}$ \\
\hline & n (\%) & n (\%) & n (\%) & n (\%) \\
\hline $\begin{array}{l}\text { Endócrinas, nutricionais e metabólicas - } \\
\text { diabetes mellitus }\end{array}$ & $17(21,0)$ & $14(17,7)$ & $58(18,0)$ & $89(18,5)$ \\
\hline $\begin{array}{l}\text { Endócrinas, nutricionais e metabólicas - } \\
\text { exceto diabetes mellitus }\end{array}$ & $07(8,6)$ & $04(5,0)$ & $11(3,4)$ & $22(4,6)$ \\
\hline Sistema osteomuscular e do tecido conjuntivo & $01(1,2)$ & $02(2,5)$ & $08(2,5)$ & $11(2,3)$ \\
\hline Outros* & $15(18,5)$ & $22(27,8)$ & $99(31,0)$ & $136(28,2)$ \\
\hline
\end{tabular}


de Petry \& Roman-Júnior, ${ }^{20}$ no qual $64 \%$ aprenderam a utilizar as plantas medicinais com os avós e pais. Segundo esses autores, o uso de plantas medicinais é baseado na tradição e a indicação e forma de utilização popular nem sempre é condizente com as apresentadas na literatura científica.

Em relação aos dados da Tabela 2, sobre a Matricaria chamomilla L., não foram encontrados estudos que comprovassem a ação antitérmica, antitussígena ou sobre os sintomas da gripe, atribuídas a esta planta pelos entrevistados. Contudo seu efeito anti-inflamatório é comprovado por estudos como o de Lins et al., ${ }^{11}$ que avaliaram efeito da $M$. recutita e da clorexidina em pacientes portadores de gengivite crônica e concluíram que a eficácia dos dois tratamentos foi equivalente.

O Formulário de Fitoterápicos da Farmacopeia Brasileira ${ }^{9}$ confirma as ações ansiolítica e sedativa leve, atribuída a Lippia alba (Mill.) N.E. Br. ex Britton \& P. Wilson pelos entrevistados e que, além dessas, apresenta ação antiespasmódica e antidispéptica. Pode ser usada ainda como emenagoga e para problemas gastrointestinais ${ }^{12}$ (Tabela 2). A indicação da planta como anti-hipertensiva pode estar relacionada à ação sedativa leve e ansiolítica, pois o estresse agudo está entre uma das causas da HAS. ${ }^{25}$ As ações antitérmica e antigripal não foram encontradas na literatura pesquisada.

Destaca-se que Plectranthus barbatus Andrews teve todas as indicações citadas pelos entrevistados confirmadas na literatura (Tabela 2), ou seja, alívio da dispepsia, ${ }^{9,13}$ e sintomas leves de desordens espasmódicas do trato gastrointestinal, com propriedades estimulantes e digestivas. ${ }^{12,13}$

Pimpinella anisum L. foi citada pelos usuários como calmante, analgésico e para problemas de estômago. Segundo Brasil, ${ }^{9}$ tem indicação como estimulante das funções digestivas, distensão abdominal e flatulência. Acredita-se que a indicação como calmante e analgésico deve-se ao fato do uso do chá promover melhoras no quadro gastrointestinal, associando a analgesia.

Verificou-se que, Mentha L., Mikania glomerata Spreng., Citrus L., Achyrocline satureioides (Lam.), Matricaria chamomilla L. e Lippia alba (Mill.) N.E. Br. ex Britton \& P. Wilson foram indicadas pelos usuários para tratamento sintomático de gripe, porém apenas o M. glomerata Spreng. tem indicação para este uso. ${ }^{9}$

A Malva sylvestris L. apresenta propriedades emolientes no tratamento de inflamações, efeito cicatrizante em feridas e potenciais nutracêuticos (propriedade antioxidante nas folhas). ${ }^{18} \dot{E}$ usada como antisséptico da cavidade oral, em bronquite, tosse, laringite, expectorante e como laxativa. As folhas apresentam propriedades hipoglicemiantes. ${ }^{9,12}$ Alguns constituintes presentes na nesta planta podem ser responsáveis pela atividade antimicrobiana. Resultados de ensaio antibacteriano indicaram que as flores e folhas de exibiram alta atividade bactericida contra alguns patógenos humanos, entre eles Staphylococcus aureus, Streptococcus agalactiae e Enterococcus faecalis. ${ }^{17}$ Estes dados oferecem evidências farmacológicas para o uso tradicional desta planta como antibacteriana.

Já a Citrus L. possui propriedades antiespasmódicas e calmantes, particularmente no tratamento de insônias, ${ }^{9,12}$ além de atividade ansiolítica e sedativa leve. ${ }^{15,16}$ Devem ser utilizadas as flores secas para preparo do chá, ${ }^{9}$ tendo sido relatado o uso de folhas pelos usuários. As indicações da planta para tratamento sintomático da gripe (Tabela 2) podem estar relacionadas com a associação que é feita desta planta com a vitamina C, pois o fruto é rico neste componente e o seu uso para este fim foi encontrado na literatura pesquisada. ${ }^{16}$

Por outro lado, no estudo de Costa $^{12}$ se atribui aos frutos de maçãs maduras propriedades refrescantes e laxativas devido ao ácido málico e aos seus sais naturais, conferindo com as indicações dos usuários entrevistados. 
A alta prevalência de portadores de HAS e DM que buscam a prevenção ou controle destas doenças crônicas com medidas como o uso de plantas medicinais, justifica-se por se tratar de uma alternativa de custo baixo e culturalmente difundida. ${ }^{26}$ Santos et al. ${ }^{27}$ em Vitória de Santo Antão/PE, mostram que dentre os 158 pacientes diabéticos entrevistados, 36,5\% referiram uso de algum tipo de planta medicinal ou fitoterápico no tratamento. Segundo Marliére et al., ${ }^{28}$ os dados relativos ao uso de plantas medicinais por hipertensos e diabéticos demonstram que esta forma de tratamento já está bastante difundida, como tratamento inicial ou complementar ao alopático, principalmente por serem doenças crônicas cujo tratamento, muitas vezes, estende-se ao longo de toda a vida do usuário.

As plantas mais citadas neste estudo para tratamento de HAS, Lippia alba (Mill.) e Mentha L., constam na RDC 10/10, porém as indicações citadas não conferem com as indicações desta referência. Eugenia uniflora L. foi citada por cinco entrevistados para o tratamento da HAS, consta na RDC 10/10 para tratamento de diarreia não infecciosa, mas, conforme Lopes, ${ }^{14}$ pode ser usada para tratar doenças do sistema cardiovascular, diminuindo a pressão arterial e para o sistema urinário, com efeito diurético. Segundo esse mesmo autor, o uso de plantas medicinais para tratamento de HAS requer embasamento científico, evitando prejuízo ao usuário, seja pela falta do controle efetivo da doença, seja por interações desconhecidas das plantas com medicamentos.

Para tratamento de DM, foram citadas Plantago major L., Zea mays ssp., Syzygium jambolanum (Lam.) DC, Campomanesia xanthocarpa Mart. ex O. Berg, Cissus sicyoides fo. lobata (Baker) Planch. e Taraxacum F.H. Wigg., porém não foi encontrada essa indicação na literatura. Destas, apenas Plantago major L e Taraxacum F.H. Wigg constam na RDC 10/10, porém suas indicações são respectivamente anti-inflamatório e antisséptico da cavidade oral, antidisparéptico (distúrbios digestivos), estimulante do apetite e diurético. ${ }^{9}$

O uso de plantas medicinais associado ao aumento da faixa etária, verificado nesse estudo, pode ser justificado pelo fato de a maioria doenças crônicas estar relacionada ao envelhecimento da população. Estas doenças exigem um nível mais complexo de assistência, o que por vezes não é ofertado de maneira ágil e integral pelo sistema público de saúde. ${ }^{29}$ Em consequência, estes indivíduos buscam terapias mais acessíveis, como o uso de plantas medicinais para o tratamento de suas doenças. Muitos mantêm o uso de plantas medicinais como forma de tratamento por costume, pelo fácil acesso e por obterem alívio para os sintomas apresentados.

Andrade et al. ${ }^{30}$ desenvolveram um estudo na comunidade Várzea Comprida dos Oliveiras, localizada no município de Pombal/PB, com 40 moradores locais. A área foi escolhida pela a comunidade apresentar um grande potencial de cultivo de plantas medicinais, por manter uma forma de vida voltada ao uso dos recursos naturais. Do total de entrevistados, $85 \%$ informaram se tratar com plantas medicinais, reforçando a ideia de que as pessoas residentes na comunidade estudada utilizam a fitoterapia como forma de tratar suas doenças, e usam as plantas medicinais também para prevenção e baseada em conhecimento popular. Para muitos usuários de plantas medicinais, a sua importância se dá ao fato de acreditarem que os recursos naturais são benéficos à saúde e também por acreditarem que o seu uso não pode prejudicar o organismo. ${ }^{31,32}$

Em relação ao predomínio do sexo feminino, demonstra a importância das mulheres na transmissão do conhecimento entre gerações, transmitindo o conhecimento sobre plantas medicinais no cuidado da saúde da família. ${ }^{21,33}$ 
Os dados relativos a escolaridade vão ao encontro de estudo realizado por Gonçalves et al., ${ }^{4}$ em Volta Redonda/RJ, composta aleatoriamente por 485 usuários de 14 UBS e da Farmácia Municipal da cidade, com objetivo de obter dados sobre o conhecimento e uso de plantas medicinais. Foi constatado que as duas faixas salariais mais baixas (44\% ganha menos que dois salários mínimos e 45,9\% entre dois e quatro salários) representavam $90 \%$ dos entrevistados que usava plantas medicinais na pesquisa.

Os dados referentes à escolaridade, renda e uso de plantas observadas neste trabalho se justificam pelo fato da amostra populacional estudada ter sido a que busca o Sistema Público de Saúde, caracterizado por indivíduos que possuem menor nível de escolaridade e poder aquisitivo, como confirmado em estudos de Brasileiro et al. ${ }^{4,34,35}$ Nesta população há índices mais elevados de procura por terapias alternativas, considerando os altos custos de medicamentos alopáticos. ${ }^{4}$ Da mesma maneira, em estudo de Ethur et al., ${ }^{36}$ realizado no município de Itaqui/RS, com 183 usuários, com objetivo de verificar o perfil dos usuários de plantas medicinais, observou-se que $85 \%$ dos que utilizavam plantas na terapêutica possuíam renda na faixa de 0 a 3 salários mínimos.

Embora o uso de plantas medicinais seja amplamente difundido, percebe-se que ainda existe escassez de informações, e a falta destas se dá principalmente entre indivíduos de nível escolar mais baixo, podendo gerar uso incorreto das mesmas, provocando agravamento das doenças já existentes ou aparecimento de novas. A cultura popular faz com que as indicações sejam transmitidas de pessoa para pessoa, atingindo principalmente os indivíduos menos favorecidos economicamente. ${ }^{37}$ Assim, os dados relativos à escolaridade e renda são imprescindíveis para se avaliar o nível de compreensão destes indivíduos sobre a necessidade de se fazer o uso correto das plantas com base em indicações comprovadas cientificamente.

Diante disso, há a necessidade de estudos comprovando a eficácia da prática do uso de plantas medicinais em seres humanos, estabelecendo, assim, o perfil de toxicidade e uso em longo prazo em diabéticos, acompanhando a evolução das complicações da doença. O conhecimento das propriedades e a correta identificação das plantas medicinais permitirá seu uso com segurança trazendo benefícios ao organismo pelas propriedades que possuem, ou diminuirá a exposição da população a práticas pouco seguras. ${ }^{24,27}$

Em 2010, o Ministério da Saúde aprovou o projeto chamado "Farmácia Viva", que propõe a inserção do uso de plantas medicinais e fitoterápicos no âmbito do SUS, ${ }^{38}$ envolvendo todas as etapas desde o cultivo, a coleta, o processamento, o armazenamento de plantas medicinais, a manipulação e a dispensação de preparações magistrais e oficinais de plantas medicinais e fitoterápicos, devendo obedecer às boas práticas de processamento e armazenamento de plantas medicinais, preparação e dispensação destes produtos. ${ }^{39}$ Novamente, reforça-se a necessidade de informações sobre eficácia e segurança para que o uso racional das plantas seja difundido, estabelecendo-se uma relação entre os saberes tradicionais e o conhecimento científico.

Para que sejam realizadas orientações corretas sobre usos e indicações terapêuticas de plantas medicinais, é necessário, juntamente com os usuários, fazer a correta identificação botânica destas plantas que fazem parte do seu cotidiano. Esta foi uma das limitações deste estudo, pois não foi realizada coleta do material vegetal, por se tratar de entrevistas realizadas em UBSs, impossibilitando averiguar o conhecimento dos indivíduos quanto à identificação correta da planta e, assim, conferindo com a sua indicação. Diante disso, fica demonstrada a possibilidade de novos estudos nessa área, dentre eles a identificação botânica 
das plantas medicinais tradicionalmente utilizadas pela população devido a grande variedade de espécies existentes e comumente utilizadas.

\section{Conclusões}

Para que possa se propagar o conhecimento e se fazer um uso seguro de plantas medicinais e fitoterápicos, os profissionais da saúde devem estar habilitados para a prescrição dos mesmos, com base em conhecimentos técnico-científicos e princípios éticos, considerando as resoluções profissionais bem como as da Anvisa, buscando, dessa maneira, atender de forma racional e qualificada às necessidades da população que buscam atendimento nas UESF. ${ }^{40}$

De acordo com o preconizado pelas políticas nacionais e pelas recomendações da Organização Mundial da Saúde, deve-se garantir à população o acesso seguro, eficaz e de qualidade, promovendo o uso racional das plantas medicinais e fitoterápicos, seja a planta medicinal in natura, seca (droga vegetal), fitoterápico manipulado ou industrializado. Isso deve ser realizado pela capacitação dos profissionais da área da saúde incorporando esta prática como recurso terapêutico, garantindo, assim, a implementação das políticas por meio das UESF. O conhecimento da fitoterapia como prática complementar no cuidado dos indivíduos é necessário para que se possa orientá-los sobre a forma adequada de identificação, preparo e usos de plantas e, desta maneira, conscientizá-los sobre o uso racional das mesmas. Numa perspectiva ampla, a fitoterapia pode e deve ser considerada como um campo de interação de saberes e práticas que valoriza os recursos culturais, práticas e saberes locais, a preservação das riquezas naturais e da biodiversidade, a interação dos usuários com a natureza e com os profissionais da equipe de saúde, além de promover a socialização da pesquisa científica e desenvolver visão crítica na população sobre o uso de plantas medicinais na APS e no setor familiar. ${ }^{41}$

\section{REFERÊNCIAS}

1. Organização Mundial de Saúde (OMS). Traditional medicine: definitions. Nota descriptiva №134 [Acesso: 19 Fev 2013]. Disponível em: http://www.who.int/medicines/areas/traditional/definitions/en/

2. Iserhard ARM, Budó MLD, Neves ET, Badke MR. Práticas culturais de cuidados de mulheres mães de recém-nascido de risco do Sul do Brasil. Esc Anna Nery Rev Enferm. 2009;13(1):116-22. DOI: http://dx.doi.org/10.1590/S1414-81452009000100016

3. Nicoletti MA, Oliveira-Júnior MSA, Bertasso CC, Caporossi PY, Tavares APL. Principais interações no uso de medicamentos fitoterápicos. Infarma. 2007;19(1/2):32-40.

4. Gonçalves NMT, Generutti M, Chaves DAS, Duarte MM, Vila C. A tradição popular como ferramenta para a implantação da fitoterapia no município de Volta Redonda - RJ. Rev Bras Farm. 2011;92(4):346-51.

5. Schwambach KH, AmadorTA. Estudo da utilização de plantas Medicinais e medicamentos em um município do Sul do Brasil. Lat Am J Pharm. 2007;26(4):602-8. http://www.lume.ufrgs.br/bitstream/handle/10183/10381/000597945.pdf?sequence=1.

6. Brasil. Instituto Brasileiro de Geografia e Estatística (IBGE). Rio Grande do Sul, ljuí, RS [Acesso: 13 Mar 2013]. Disponível em: http://www.ibge.gov.br/cidadesat/painel/painel.php?codmun=431020

7. Brasil. Ministério da Saúde. Série B. Textos Básicos de Saúde. Brasília-DF [Acesso: 17 Jun 2013]. Disponível em: http:// conselho.saude.gov.br/biblioteca/Relatorios/plano_nacional_saude_2012_2015.pdf

8. Brasil. Agência Nacional de Vigilância Sanitária. Resolução - RDC no 10, de 9 de março de 2010. Dispõe sobre a notificação de drogas vegetais junto à Agência Nacional de Vigilância Sanitária (ANVISA) e dá outras providências. Diário Oficial da República Federativa do Brasil, Poder Executivo, Brasília, DF, 10 mar. 2010. Seção 1, p. 52-59. (2010) [Acesso 15 Jun 2013]. Disponível em: http://189.28.128.100/dab/docs/legislacao/resolucao10_09_03_10.pdf 
9. Brasil. Agência Nacional de Vigilância Sanitária. Formulário de Fitoterápicos da Farmacopéia Brasileira/Agência Nacional de Vigilância Sanitária. Brasília: Anvisa. p.126. (2011b) [Acesso 15 Jun 2013]. Disponível em: http://www.anvisa.gov.br/ hotsite/farmacopeiabrasileira/arquivos/2012/FNFB\%202_Revisao_2_COFAR_setembro_2012_atual.pdf

10. Simões, CMO, Mentz LA, Schenkel EP, Irgang BE, Stehmann JR. Plantas da medicina popular no Rio Grande do Sul. 6a ed. Porto Alegre: Universidade Federal do Rio Grande do Sul; 2004.

11. Lins R, Vasconcelos FHP, Leite RB, Coelho-Soares RS, Barbosa DN. Avaliação clínica de bochechos com extratos de Aroeira (Schinus terebinthifolius) e Camomila (Matricaria recutita L.) sobre a placa bacteriana e a gengivite. Rev Bras Plantas Med. 2013;15:(1)112-20. DOI: http://dx. doi.org/10.1590/S1516-05722013000100016

12. Costa AF. Farmacognosia. 6a ed. Lisboa: Fundation Calouste Gulbenkian; 2002. p.1031.

13. EMEA. CommunityherbalmonographonPeumusboldusmolinafolium. EMEA/HMPC [Acesso 05 Jun 2013]. Disponível em: http://www.ema.europa.eu/

14. Lopes GAD, Feliciano LM, Diniz RES, Alves MJQF. Plantas medicinais: indicação popular de uso no tratamento de hipertensão arterial sistêmica (HAS). Rev Cien Ext. 2010;6:(2)143-55.

15. Costa CA, Cury TC, Cassettari BO, Takahira RK, Flório JC, Costa M. Citrus aurantium L. essential oil exhibits anxiolytic-like activity mediated by $5-\mathrm{HT}(1 \mathrm{~A})$-receptors and reduces cholesterol after repeated oral treatment. BMC Complement Altern Med. 2013;13:42. DOI: http://dx.doi.org/10.1186/1472-6882-13-42

16. Oliveira IP, Oliveira LC, Moura CSFT. Frutas cítricas. Rev Fac Montes Belos. 2012;5(4):78-94.

17. Razavi SM, Zarrini G, Molavi G, Ghasemi G. Bioactivity of malva sylvestris L., a medicinal plant from Iran. Iran J Basic Med Sci. 2011;14(6):574-9.

18. Pirbalouti AG, Azizi S, Koohpayeh A. Healing potential of Iranian traditional medicinal plants on burn wounds in alloxaninduced diabetic rats. Rev Bras Farmacogn. 2012;22(2)397-403. DOI: http://dx.doi.org/10.1590/S0102-695X2011005000183

19. Oliveira Junior RG, Lavor EM, Oliveira MR, Souza EV, Silva MA, Silva MTNM, Nunes LMN. Plantas medicinais utilizadas por um grupo de idosos do município de Petrolina, Pernambuco. Rev Eletron Farm. 2012;9(3):16-28.

20. Petry K, Roman Júnior WA. Viabilidade de implantação de fitoterápicos e plantas medicinais no Sistema Único de Saúde (SUS) do município de Três Passos/RS. Rev Bras Farm. 2012;93(1):60-7.

21. Lima ARA, Vasconcelos MKP, Barbieri RL, Heck RM. Plantas medicinais utilizadas pelos octogenários e nonagenários de uma vila periférica de Rio Grande/RS, Brasil. Rev Enferm UFPE. 2011;5(5):329-36.

22. Hughes GD, Aboyade OM, Clark BL, Puoane TR. The prevalence of traditional herbal medicine use among hypertensives living in South African communities. BMC Complement Altern Med. 2013;13:38. http://dx.doi.org/10.1186/1472-6882-1338. DOI: http://dx.doi.org/10.1186/1472-6882-13-38

23. Costa JR, Mitja D. Uso dos recursos vegetais por agricultores familiares de Manacapuru (AM). Acta Amaz. 2010;40(1):4958. DOI: http://dx.doi.org/10.1590/S0044-59672010000100007

24. Silva AG. Uso, conservação e diversidade de plantas aromáticas, condimentares e medicinais para fins medicinais na comunidade Vila Princesa, Porto Velho - RO. Rev Pesq Criac. 2011;10(2):21-35.

25. Brasil. Ministério da Saúde. Cadernos de Atenção Básica n. 15. Hipertensão Arterial Sistêmica. Brasília, DF. 2006 [Acesso 14 Ago 2015]. Disponível em: http://bvsms.saude.gov.br/bvs/publicacoes/caderno_atencao_basica15.pdf

26. Oliveira CJ, Araújo TL. Plantas medicinais: usos e crenças de idosos portadores de hipertensão arterial. Rev Eletron Farm. 2007;9(1):93-105.

27. Santos MM, Nunes MGS, Martins RD. Uso empírico de plantas medicinais para tratamento de diabetes. Rev Bras Plantas Med. 2012;14(2):327-34. DOI: http://dx.doi.org/10.1590/S1516-05722012000200012

28. Marliére LDP, Ribeiro AQ, Brandão MGL, Klein CH, Acurcio FA. Utilização de fitoterápicos por idosos: resultados de um inquérito domiciliar em Belo Horizonte (MG), Brasil. Rev Bras Farmacogn. 2008. 18(Supl.):754-60.

29. Veras R. Envelhecimento populacional contemporâneo: demandas, desafios e inovações. Rev Saúde Pública. 2009;43(3):548-54. DOI: http://dx.doi.org/10.1590/S0034-89102009000300020

30. Andrade SEO, Maracajá PB, Silva RA, Freires GF, Pereira AM. Estudo etnobotânico de plantas medicinais na comunidade Várzea Comprida dos Oliveiras, Pombal, Paraíba, Brasil. Rev Verde Agroecol Desenvolv Sustent. 2012;7(3):46-52. 
31. Badke MR, Budó MLD, Silva FM, Ressel LB. Plantas medicinais na prática do cotidiano popular. Esc Anna Nery Rev Enferm. 2011;15(1):132-9. DOI: http://dx.doi.org/10.1590/S1414-81452011000100019

32. Oliveira ER, Menini Neto L. Levantamento etnobotânico de plantas medicinais utilizadas pelos moradores do povoado de Manejo, Lima Duarte - MG. Rev Bras Plantas Med. 2012;14(2):311-20. DOI: http://dx.doi.org/10.1590/S151605722012000200010

33. Ceolin T, Hech RM, Barbieri RL, Schwartz E, Muniz RM, Pillon CN 2011. Plantas medicinais: transmissão do conhecimento nas famílias de agricultores de base ecológica no Sul do RS. Rev Esc Enferm USP. 2011;45(1):47-54. DOI: http://dx.doi. org/10.1590/S0080-62342011000100007

34. Brasileiro BG, Pizziolo VR, Matos DS, Germano AM, Jamal CM. Plantas medicinais utilizadas pela população atendida no "Programa de Saúde da Família", Governador Valadares, MG, Brasil. Rev Bras Ciênc Farm. 2008;44(4):629-36. DOI: http://dx.doi.org/10.1590/S1516-93322008000400009

35. Suassuna JM. O uso de plantas medicinais pela população na Unidade Básica de Saúde da Família (UBSF). Trabalho de Conclusão de Curso (Graduação em Enfermagem). Universidade Estadual da Paraíba. Centro de Ciências Biológicas e da Saúde. Campina Grande; 2011.

36. Ethur LZ, Jobim JC, Ritter JG, Oliveira G, Trindade BS. Comércio formal e perfil de consumidores de plantas medicinais e fitoterápicos no município de Itaqui - RS. Rev Bras Plantas Med. 2011;13(2):121-8.

37. Macedo AF, Oshiiwa M, Guarido CF. Ocorrência do uso de plantas medicinais por moradores de um bairro do município de Marília - SP. Rev Ciênc Farm Básica Apl. 2007;28(1):123-8.

38. Brasil. Ministério da Saúde. Portaria № 886, de 20 de abril de 2010. Institui a Farmácia Viva no âmbito do Sistema Único de Saúde; 2010.

39. Brasil, Ministério da Saúde. RDC № 18, de 3 de abril de 2013 Dispõe sobre os produtos magistrais e oficinais de plantas medicinais e fitoterápicos em farmácias vivas no âmbito do Sistema Único de Saúde; 2013.

40. Brasil. Conselho Federal de Farmácia (CFF). Resolução - RDC no 546 de 21 de julho de 2011. Dispõe sobre a indicação farmacêutica de plantas medicinais e fitoterápicos isentos de prescrição e o seu registro. Brasília, DF. Diário Oficial da União de 26/07/2011, Seção 1, Página 87; 2011.

41. Antonio GD, Tesser CD, Moretti-Pires RO. Contributions of medicinal plants to care and health promotion in primary healthcare. Interface (Botucatu). 2013;17(46):615-33. DOI: http://dx.doi.org/10.1590/S1414-32832013005000014 